\title{
Nanocompósitos de Blendas HDPE/LLDPE e OMMT - Parte I: Avaliação das Propriedades Termo-mecânicas e da Resistência ao Intemperismo
}

\author{
Fabio R. Passador, Eduardo H. Backes, Daniel R. Travain, Adhemar Ruvolo Filho, Luiz A. Pessan \\ Departamento de Engenharia de Materiais, UFSCar
}

\begin{abstract}
Resumo: Nanocompósitos de blendas de polietileno de alta densidade (HDPE) com polietileno linear de baixa densidade (LLDPE) e OMMT (montmorilonita organofílica) foram preparados sob fusão em extrusora de duplarosca, utilizando HDPE-g-MA como agente compatibilizante. A caracterização estrutural foi realizada por análises de difração de raios X em alto ângulo (WAXD), microscopia eletrônica de transmissão (MET). Os resultados indicam que a adição do agente compatibilizante favoreceu a formação da microestrutura predominantemente intercalada. Estudos dinâmico-mecânicos mostraram que a adição do agente compatibilizante aumentou as interações entre a carga e a matriz poliolefínica. As diferentes condições de intemperismo as quais os materiais foram submetidos influenciaram no comportamento mecânico dos nanocompósitos de blenda HDPE/LLDPE. Os tratamentos realizados em estufa com circulação forçada de ar e em água proporcionaram o alívio de tensões residuais presentes no sistema, enquanto que o tratamento realizado em câmara de envelhecimento acelerado levou à formação de grupos carbonílicos, de pequena massa molar, que diminuíram o grau de cristalinidade e o módulo elástico dos nanocompósitos.
\end{abstract}

Palavras-chave: Nanocompósitos, HDPE/LLDPE, intemperismo.

\section{HDPE/LLDPE Blend-based Nanocomposites - Part I: Evaluation of Thermo-mechanical Properties and Weathering Resistance}

\begin{abstract}
Nanocomposites from high density polyethylene/ linear low density polyethylene (HDPE/LLDPE) blends were prepared at the melt state in an extruder, using HDPE-g-MA as compatibilizer agent. The structural characterization was performed through wide angle X-ray diffraction (WAXD) and transmission electron microscopy (TEM). The results showed that adding the compatibilizer induced formation of a predominant intercalated microstructure. Dynamic-mechanical studies showed that the addition of the compatibilizer increases the interactions between the nanoclay surface and the polyolefin matrix. The weathering conditions affected the mechanical behavior of HDPE/LLDPE blend-based nanocomposites. Both treatments performed in hot water and in a forced convection air oven provided the relief of residual stresses in the polymer matrix, while the treatment in an accelerated aging chamber provided the formation of carbonyl groups that lead to a decreased degree of crystallinity and elastic modulus of the nanocomposites.
\end{abstract}

Keywords: Nanocomposites, HDPE/LLDPE, weathering.

\section{Introdução}

Os polietilenos são poliolefinas amplamente utilizadas em aplicações para o setor de embalagens, no setor automotivo e elétrico, onde as propriedades de resistência térmica, mecânica e de permeação a gases possuem grande importânciaa ${ }^{[1,2]}$. Nessas aplicações, usualmente o polietileno é exposto ao intemperismo e a solicitações mecânicas ${ }^{[3,4]}$.

Dentre as alternativas utilizadas para melhorar o desempenho térmico e mecânico das poliolefinas, a dispersão de camadas de silicatos lamelares tem se mostrado bastante promissora. Estudos com nanocompósitos de poliolefinas têm mostrado melhoras no desempenho das propriedades elétricas, mecânicas, retardamento de chama, flamabilidade e propriedades de barreira, com adição de quantidades de até $5 \%$ em massa de nanocargas, que é decorrente das fortes interações na interface polímero/ reforço e da elevada razão de aspecto das nanocargas ${ }^{[5-9]}$.
O grande desafio para obtenção de nanocompósitos de polietilenos com silicato lamelares é decorrente do baixo nível de interações entre a matriz poliolefínica hidrofóbica e apolar com a superfície polar dos silicatos lamelares. Muitos trabalhos ${ }^{[6-13]}$ reportam que essa situação pode ser amenizada realizando-se um tratamento na superfície dos silicatos lamelares com íons alquilamônio e utilização de agentes compatibilizantes enxertados com anidrido maleico na matriz poliolefínica.

Blendas de polietileno de alta densidade (HDPE)/ polietileno linear de baixa densidade (LLDPE) possuem grande interesse tecnológico e científico, principalmente para o uso em dielétricos compósitos, que são expostos ao oxigênio e radiação solar ultravioleta (UV). Quando em uso, a fotooxidação resultante das condições de intemperismo pode diminuir significativamente o tempo de vida útil do material. A degradação por fotólise ou por 
fotooxidação requer absorção de UV com comprimento de onda superior a $290 \mathrm{~nm}$. O polietileno fotodegrada mais rapidamente no ar que em ambiente inerte, indicando que a fotooxidação antecede a fotólise. Embora o polietileno não absorva no comprimento de onda acima de $290 \mathrm{~nm}$, o processo oxidativo radicalar poderá ser iniciado pela absorção de luz solar ${ }^{[14]}$. É importante ressaltar que o polietileno absorve fortemente a radiação UV com comprimentos de onda menores que $250 \mathrm{~nm}$, o que é bem inferior ao limite de comprimento de onda que atinge a superfície terrestre, no entanto defeitos do processo da síntese do polietileno, como duplas ligações, podem atuar como grupos cromóforos iniciando a fotodegradação. Outros efeitos decorrentes da degradação podem ser o aumento da cristalinidade durante a fotodegradação ${ }^{[15,16]}$, degradação térmica ${ }^{[17]}$, e irradiação de alta energia ${ }^{[18,19]}$; o decréscimo na cristalinidade principalmente durante a irradiação gama ${ }^{[20]}$, uma modificação na morfologia, como destruição de esferulitos ${ }^{[21]}$ e alteração na rede cristalina ${ }^{[22]}$.

A fotodegradação em HDPE e LLDPE gera preferencialmente ramificações, ao contrário do PP e LDPE, onde a cisão- $\beta$ é mais efetiva ${ }^{[23]}$. A Figura 1 apresenta o mecanismo de reticulação e de cisão- $\beta$ em poliolefinas.

A presença de ramificações e de carbonos terciários nas cadeias das poliolefinas as torna susceptíveis ao ataque por radicais livres e oxigênio e, portanto, à degradação ${ }^{[24]}$. A fotodegradação do HDPE e LLDPE pode afetar significativamente as propriedades físico-químicas dos polímeros, tais como aumento da fração cristalina, no módulo elástico e nas propriedades de barreira ${ }^{[25]}$. No entanto, a adição de cargas às poliolefinas pode afetar a sua resistência à fotooxidação ${ }^{[26,27]}$, principalmente no caso das argilas naturais que possuem alto nível de ferro em sua composição, que atuam como catalisadores dos processos fotooxidativos ${ }^{[28]}$. O objetivo principal deste trabalho foi avaliar a influência da ação do intemperismo nas propriedades mecânicas de nanocompósitos de blenda HDPE/LLDPE com diferentes teores de OMMT.

\section{Experimental}

\section{Materiais}

O polietileno de alta densidade (HDPE) de especificação DMDA 6200 NT-7 foi fornecido pela Dow Chemical, possui índice de fluidez de $0,38 \mathrm{~g} / 10 \mathrm{~min}$ $\left(190^{\circ} \mathrm{C} / 2,16 \mathrm{~kg}\right)$ e densidade de $0,953 \mathrm{~g} / \mathrm{cm}^{3}$. O polietileno linear de baixa densidade (LLDPE) de especificação

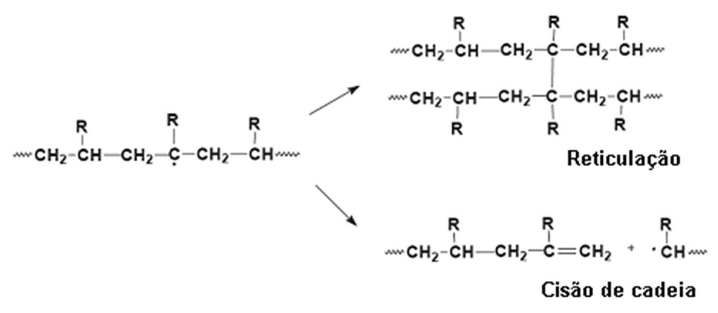

Figura 1. Ilustração do mecanismo de reticulação e de cisão- $\beta$ em poliolefinas ${ }^{[23]}$.
IC-32 foi fornecido pela Braskem, possuindo índice de fluidez de $29 \mathrm{~g} / 10 \mathrm{~min}\left(190{ }^{\circ} \mathrm{C} / 2,16 \mathrm{~kg}\right)$ e densidade de $0,924 \mathrm{~g} / \mathrm{cm}^{3}$. O agente compatibilizante utilizado foi o polietileno de alta densidade enxertado com anidrido maleico (HDPE-g-MA) de especificação Polybond ${ }^{\circledR}$ 3009, da Crompton Corporation, com índice de fluidez de $3 \mathrm{~g} / 10 \mathrm{~min}\left(190{ }^{\circ} \mathrm{C} / 2,16 \mathrm{~kg}\right)$ e funcionalizado com $1 \%$ em massa de anidrido maleico. Para obtenção dos nanocompósitos foi utilizada a montmorilonita natural modificada com um sal quaternário de amônio (OMMT), de nome comercial Cloisite ${ }^{\circledR} 20 \mathrm{~A}$ produzida pela Southern Clay Products Inc., com densidade aparente de aproximadamente $0,32 \mathrm{~g} / \mathrm{cm}^{3}$, com perda de massa durante a queima de $38 \%$ e com espaçamento basal de $2,32 \mathrm{~nm}$. A argila Cloisite ${ }^{\circledR} 20 \mathrm{~A}$ é tratada com surfactante apolar e possui alto grau de hidrofobicidade. O teor de nanocarga adicionada nos sistemas foi corrigido considerando o teor de material orgânico na mesma.

\section{Preparação dos nanocompósitos}

A blenda HDPE/LLDPE e os nanocompósitos foram preparados a partir da mistura no estado fundido em uma extrusora dupla rosca co-rotacional MT19TC da marca B\&P Process Equipament and Systems com diâmetro de $19 \mathrm{~mm}$ e L/D = 25 com velocidade das roscas a $120 \mathrm{rpm}$, vazão de $0,7 \mathrm{~kg} / \mathrm{h}$ e com um perfil de temperatura de $180{ }^{\circ} \mathrm{C} / 190{ }^{\circ} \mathrm{C} / 190{ }^{\circ} \mathrm{C} / 200{ }^{\circ} \mathrm{C} / 210^{\circ} \mathrm{C}$.

Os nanocompósitos de blenda HDPE/LLDPE e OMMT com a adição de HDPE-g-MA como agente compatibilizante foram preparados em duas etapas. $\mathrm{Na}$ primeira etapa de mistura foi preparado o nanocompósito de HDPE/HDPE-g-MA/OMMT e numa segunda etapa de mistura foi adicionado o LLDPE. A razão de HDPE/ LLDPE utilizada foi de 3:1 e a razão de OMMT/HDPE-gMA foi de 1:2. Foram estudados três teores de OMMT: 2,5; 5,0 e $7,5 \%$ em massa e que foram designados por $\mathrm{NC}(2,5)$, $\mathrm{NC}(5,0)$ e $\mathrm{NC}(7,5)$, respectivamente. Nanocompósitos de blenda HDPE/LLDPE e OMMT sem adição de agente compatibilizante foram preparados em uma única etapa, com razão HDPE/LLDPE de 3:1 e três teores de OMMT: 2,$5 ; 5,0$ e 7,5\% em massa e foram designados por $\mathrm{NSC}(2,5), \operatorname{NSC}(5,0)$ e $\mathrm{NSC}(7,5)$, respectivamente. Os materiais puros e a blenda HDPE/LLDPE (75/25\%) também foram preparados nas mesmas condições e seus resultados foram utilizados para efeitos comparativos.

\section{Conformação dos corpos de prova através de moldagem por injeção}

Corpos de prova para ensaios de tração uniaxial (tipo IV com formato de "gravata") foram conformados utilizando uma injetora modelo $270 \mathrm{~V}$ da marca Arburg Allrounder com perfil de temperaturas de $225{ }^{\circ} \mathrm{C} / 230$ ${ }^{\circ} \mathrm{C} / 235{ }^{\circ} \mathrm{C} / 240{ }^{\circ} \mathrm{C} / 240{ }^{\circ} \mathrm{C}$. A pressão de injeção e as contrapressões utilizadas foram de 1900 bar e 120 bar, respectivamente. Outras condições utilizadas foram velocidade da rosca de $12 \mathrm{~m} / \mathrm{min}$, temperatura do molde a $45^{\circ} \mathrm{C}$ e tempo de resfriamento de 45 segundos.

\section{Caracterização estrutural}

O grau de intercalação/esfoliação da nanoargila e dos nanocompósitos foi avaliado através de análises 
de difração de Raios-X de alto ângulo (WAXD) e microscopia eletrônica de transmissão (MET).

Análises de WAXD foram realizadas utilizando-se um difratômetro Rigaku Rotaflex, com radiação $\mathrm{CuK} \alpha$ $(\lambda=1,54056 \AA)$, operado a $40 \mathrm{KV}$ e $80 \mathrm{~mA}$, com $2 \theta$ variando entre 1,5 e $10^{\circ}$, a uma taxa de $1 \%$ min.

A morfologia dos nanocompósitos foi analisada pela observação de imagens obtidas em um microscópio eletrônico de transmissão. As amostras foram submetidas a "trimming" (corte na forma de trapézio) e foram cortadas em seções ultrafinas, $40 \mathrm{~nm}$ de espessura, usando-se um micrótomo tipo Riechert-Jung Ultracut E, com faca de diamante, com temperatura da amostra entre -65 e $-75{ }^{\circ} \mathrm{C}$ e temperatura da faca em $-50{ }^{\circ} \mathrm{C}$. Foram utilizadas telas ou "grids" de cobre para coletar as amostras fatiadas que se encontravam imersas em uma solução de DMSO:água (3:2). Posteriormente, as amostras foram analisadas em microscópio eletrônico de transmissão Philips, modelo CM120, a uma voltagem de aceleração de $120 \mathrm{KV}$.

\section{Análise dinâmico-mecânica}

A análise dinâmico-mecânica foi realizada em um equipamento DMA da TA Instruments, modelo Q800. O equipamento DMA foi operado no modo flexão em três pontos, a uma frequência de $1 \mathrm{~Hz}$, taxa de aquecimento de $2{ }^{\circ} \mathrm{C} / \mathrm{min}$ e intervalo de temperatura de -130 a $110{ }^{\circ} \mathrm{C}$.

\section{Temperatura de Deflexão Térmica (HDT)}

$\mathrm{O}$ ensaio de HDT foi realizado em um equipamento da CEAST HDT 6 VICAT, P/N 6921.0009001 de acordo com a norma ASTM D 648-07 ${ }^{[42]}$, com taxa de aquecimento de $120{ }^{\circ} \mathrm{C} / \mathrm{h}$ até que o corpo defletisse $0,25 \mathrm{~mm}$ sob uma tensão de $450 \mathrm{kPa}$.

\section{Tratamento térmico dos nanocompósitos}

Os corpos de prova para ensaio de tração foram submetidos a três tipos de tratamento para simulações das condições de intemperismo natural no clima tropical.

E0: corpos de prova de referência, sem tratamento;

E1: envelhecimento em estufa com circulação forçada de ar, à temperatura de $110^{\circ} \mathrm{C}$, por um período de 7 dias;

E2: envelhecimento por imersão em água, à temperatura de $60{ }^{\circ} \mathrm{C}$, por um período de 7 dias. O envelhecimento em água foi realizado imergindo-se os corpos de prova dentro de um balão volumétrico de $2000 \mathrm{~mL}$ contendo água destilada com sistema de refluxo e aquecimento via manta térmica;

E3: envelhecimento em câmara de radiaçãoultravioleta por um período de 360 horas. O envelhecimento foi realizado em um equipamento Atlas Weather-Ometer, modelo 65 XW-WR1, com lâmpada de xenônio de $6500 \mathrm{~W}$, com filtros internos e externos de borossilicato. $\mathrm{O}$ ciclo de envelhecimento acelerado ocorreu a cada 120 minutos, com 102 minutos de insolação e 18 minutos de insolação e simulação de chuva. O ensaio foi realizado de acordo com a norma ASTM G $155-00^{[43]}$. O tempo de ensaio de 360 horas de envelhecimento no Atlas WeatherOmeter corresponde a aproximadamente 4 meses de envelhecimento natural, sem interferência de solicitações mecânicas, em local com 140 kLy (quilocaloria por centímetro quadrado ou quilolangley) de insolação anual.

\section{Ensaio de tração uniaxial}

Após cada processo de envelhecimento as diferentes composições foram submetidas a ensaios de tração uniaxial em uma máquina universal de ensaios Instron modelo 5569, com uma célula de carga de $50 \mathrm{kN}$, velocidade da travessa de $5 \mathrm{~mm} / \mathrm{min}$ com a utilização de um extensômetro.

\section{Calorimetria exploratória Diferencial das Amostras Tratadas (DSC)}

A avaliação da influência dos processos de envelhecimento no grau de cristalinidade foi determinada através de ensaios de calorimetria exploratória diferencial (DSC) dos materiais puros e da blenda HDPE/LLDPE. Para as análises utilizou-se o equipamento da TA Instruments, modelo QS100 com nitrogênio como gás de arraste, em fluxo contínuo de $50 \mathrm{~mL} \cdot \mathrm{min}^{-1}$. As amostras foram inicialmente aquecidas até $200{ }^{\circ} \mathrm{C}$ a uma taxa de aquecimento de $10{ }^{\circ} \mathrm{C} / \mathrm{min}$, permanecendo nesta temperatura por 3 minutos. A seguir foram resfriadas até $40{ }^{\circ} \mathrm{C}$ a uma taxa de $10{ }^{\circ} \mathrm{C} / \mathrm{min}$ para determinação da temperatura de cristalização $\left(\mathrm{T}_{\mathrm{c}}\right)$ e novamente foram aquecidas até $200^{\circ} \mathrm{C}$ a uma taxa de $10^{\circ} \mathrm{C} / \mathrm{min}$.

\section{Resultados e Discussão}

\section{Caracterização estrutural}

A Figura 2 apresenta os resultados das análises por WAXD da nanoargila e dos nanocompósitos com diferentes teores de OMMT.

Analisando os difratogramas dos nanocompósitos sem adição de agente compatibilizante da Figura 2a observa-se um pequeno deslocamento do pico de difração para ângulos menores referentes ao plano cristalográfico $\left(\begin{array}{lll}0 & 0 & 1\end{array}\right)$ em relação a OMMT, indicando um pequeno aumento no espaçamento basal $\left(2 \theta=3,8^{\circ}\right.$ que corresponde a um espaçamento basal de $2,32 \mathrm{~nm}$, enquanto que $2 \theta=3,3^{\circ}$ corresponde a um espaçamento basal de 2,68 nm), sugerindo que uma expressiva fração de tactóides permanece integra no sistema sem alteração da distancia entre lamelas de argila, ou seja, o cisalhamento decorrente da mistura em estado fundido não foi suficiente para que um eficiente processo de delaminação do argilomineral. Há também uma reflexão secundária para $2 \theta=7,4^{\circ}$ que corresponde ao plano cristalográfico (l $\left.\begin{array}{lll}0 & 0 & 2\end{array}\right)$, que permanece nos nanocompósitos porém se desloca para ângulos menores. No caso da adição de agente compatibilizante (Figura $2 b$ ) nota-se que o pico de difração referente ao plano cristalográfico (l $\left.\begin{array}{lll}0 & 0 & 1\end{array}\right)$ sofreu deslocamento mais significativo para menores ângulos $2 \theta$, o que corresponde a um aumento no espaçamento interlamelar do argilomineral, sugerindo que ocorreu um processo de intercalação das cadeias poliméricas entre as camadas do silicato lamelar. Com um aumento do teor de nanocarga na matriz polimérica observa-se um aumento do espaçamento basal, sendo obtido valores de $2,96 \mathrm{~nm}$ para $\mathrm{NC}(2,5), 3,04 \mathrm{~nm}$ para $\mathrm{NC}(5,0)$ e 3,17 nm para $\mathrm{NC}(7,5)$. 


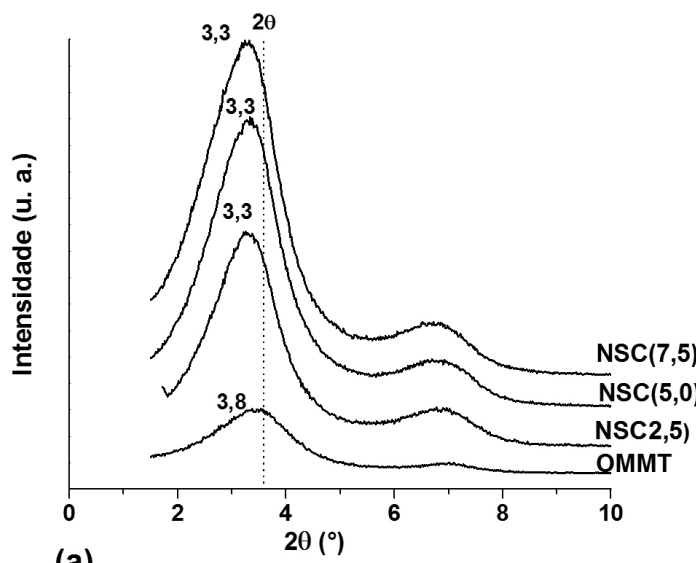

(a)

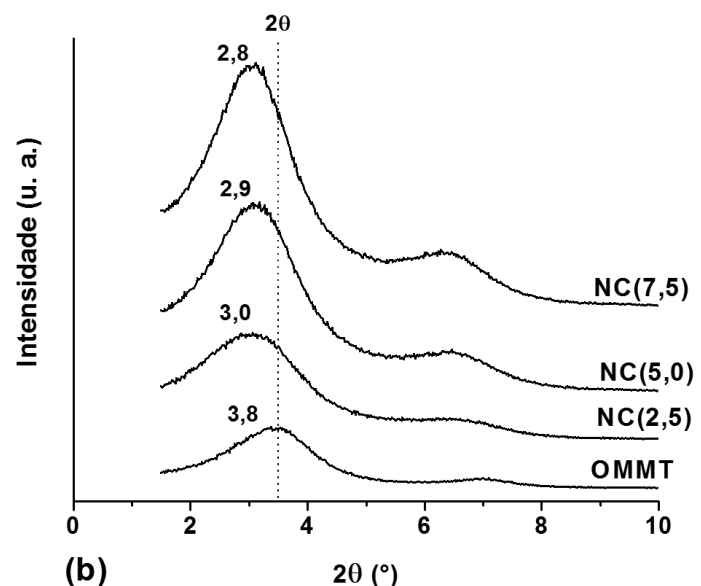

Figura 2. Difratogramas de raios-X da nanoargila e dos nanocompósitos (a) sem adição de agente compatibilizante e (b) com adição de HDPE-g-MA e diferentes teores de nanoargila.

A morfologia e o estado de dispersão da argila nos nanocompósitos foram avaliados através de imagens obtidas por microscopia eletrônica de transmissão (MET). A Figura 3 apresenta as micrografias dos nanocompósitos sem adição de agente compatibilizante.

A estrutura dos nanocompósitos é composta basicamente por tactóides da argila. A baixa interação entre os componentes (matriz e carga) não favoreceu a quebra dos tactóides durante o processamento, porém observa-se que estes estão bem dispersos na matriz polimérica. Quanto maior o teor da carga inorgânica utilizado, mais visíveis e maiores os aglomerados observados na matriz polimérica.

A Figura 4 apresenta as micrografias dos nanocompósitos compatibilizados com HDPE-g-MA e diferentes teores de OMMT.

Analisando as micrografias pode-se observar que a adição do HDPE-g-MA, com alta viscosidade, juntamente com o cisalhamento imposto no processo de extrusão facilitou o processo de quebra e redução dos tamanhos de tactóides de argila organofílica e, consequentemente, no processo de intercalação das cadeias poliméricas entre as camadas de silicato e na dispersão do argilomineral na
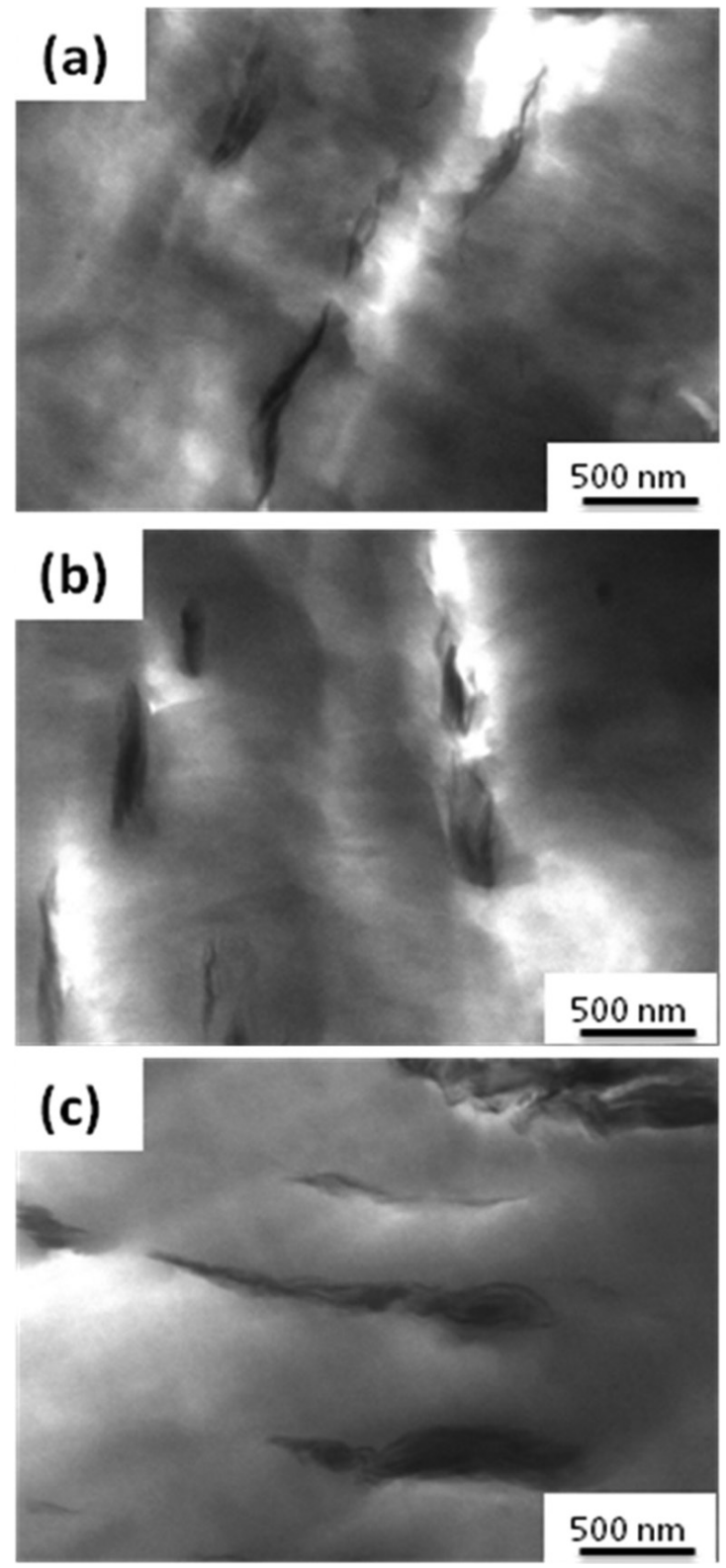

Figura 3. Micrografias obtidas por MET: (a) $\mathrm{NSC}(2,5)$, (b) $\operatorname{NSC}(5,0)$ e (c) NSC $(7,5)$. Aumento de 31.000x.

matriz polimérica. A morfologia observada nestes sistemas é composta basicamente por lamelas intercaladas, porém nota-se também a presença de algumas lamelas esfoliadas de argila organofílica e também a presença de pequenos tactóides com tamanho bastante reduzido em comparação com as morfologias observadas para os nanocompósitos sem agente compatibilizante (Figura 3).

\section{Caracterização termo-mecânica}

A Figura 5 apresenta os gráficos de módulo de perda (E”) e módulo de armazenamento (E') dos materiais puros HDPE, LLDPE e da blenda HDPE/LLDPE (75/25 \%) obtidos da análise dinâmico-mecânica. 

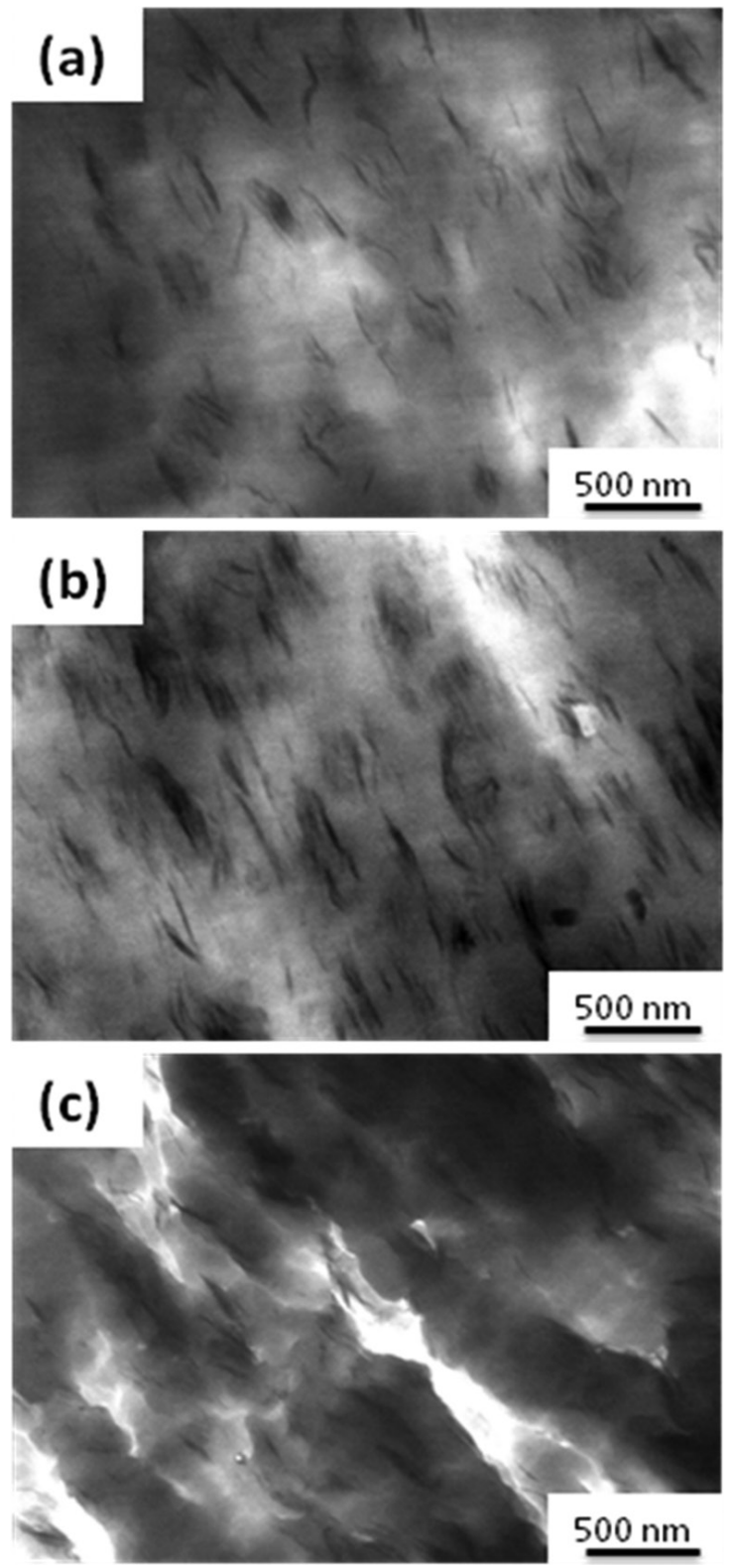

Figura 4. Micrografias obtidas por MET: (a) $\mathrm{NC}(2,5)$, (b) $\mathrm{NC}(5,0)$ e (c) NC(7,5). Aumento de 31.000x.

A relaxação molecular nos polietilenos é fortemente influenciada por variáveis que descrevem a estrutura do material, como cristalinidade, espessura lamelar e espessura da camada amorfa. A Figura 5a mostra que, a partir do resfriamento, os processos de relaxação $\alpha, \beta$ e $\gamma$ são observados, respectivamente. O processo de relaxação $\alpha$ é observado em um intervalo que vai da temperatura ambiente à temperatura de fusão; a transição $\beta$ é vista num intervalo de $0^{\circ} \mathrm{C}$ a $-50{ }^{\circ} \mathrm{C}$, aproximadamente; por fim, a transição $\gamma$ é observada no intervalo que vai de $-90{ }^{\circ} \mathrm{C} \mathrm{a}-140{ }^{\circ} \mathrm{C}$.

A relaxação $\alpha$ é observada, em geral, em todos polímeros semicristalinos e seu sinal é intensificado com o aumento da cristalinidade do material sendo por isso
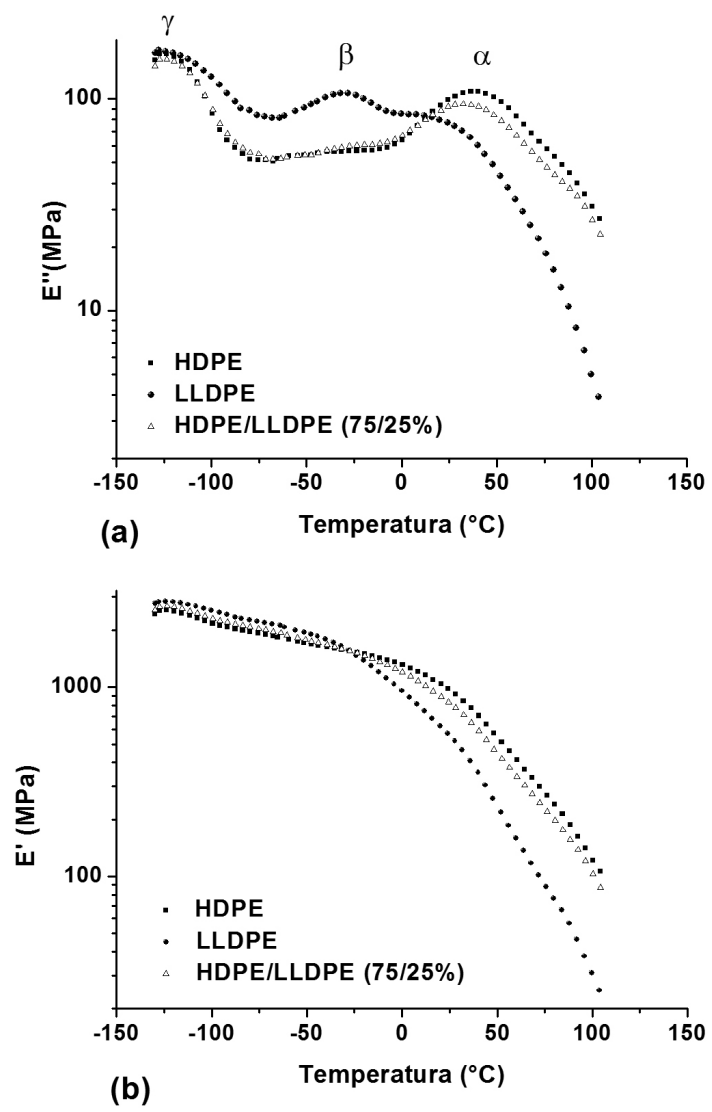

Figura 5. (a) Módulo de Perda (E") e (b) Módulo de Armazenamento (E') em função da temperatura dos materiais puros e da blenda polimérica HDPE/LLDPE.

relacionada com a mobilidade das unidades das cadeias poliméricas dentro da fase cristalina. Muitos autores ${ }^{[29-32]}$ demonstraram que a transição $\alpha$ em polietilenos consiste na verdade em dois picos superpostos designados como relaxações $\alpha_{1}$ e $\alpha_{2}$ em ordem decrescente de temperatura, onde a primeira é relacionada a um processo de escorregamento intralamelar (ou fenômeno de contorno de grão) e/ou mobilidade da região intercristalina e a segunda relacionada à mobilidade intracristalina que envolve o movimento translacional de segmentos de cadeia ao longo do eixo c dentro da célula unitária.

Com relação às transições $\beta$ e $\gamma$ há muitas interpretações conflitantes na literatura com respeito a suas origens e naturezas moleculares. Pechhold et al. ${ }^{[33]}$ e Moore et al. [34] observaram um processo fraco de relaxação em polietilenos de alto peso molecular sem ramificações na região da transição $\beta$; em contraste, esta mesma transição é bastante pronunciada para polietilenos ramificados, o que pode ser observado no presente trabalho comparando-se as curvas da Figura 5a para o HDPE e LLDPE. Kline et al. ${ }^{[35]}$ propôs que a transição $\beta$ está associada com a relaxação de ramificações curtas na fase amorfa enquanto Mandelkern et al. ${ }^{[36]}$ demonstraram que esta mesma transição resulta de processos de relaxação de segmentos de cadeia na região interfacial.

Com relação a transição $\gamma$, Wada et al. ${ }^{[37]}$ e Fischer et al. ${ }^{[38]}$ relacionaram esta ao movimento de 
segmentos de cadeia desordenados na superfície dos cristais poliméricos. Além disso, Wollbourn ${ }^{[39]}$ mostrou que a transição $\gamma$ se relaciona a transição vítrea da sequência metilênica e tem o mesmo efeito da transição frágil-dúctil. Estes investigadores acreditam que este processo de relaxação corresponde à transição vítrea do polietileno, porém apesar de extensiva pesquisa nesta área, não há uma aceitação unânime para qualquer explicação da origem molecular da transição $\gamma$.

A Figura 5b por sua vez, mostra que, a baixas temperaturas o LLDPE apresenta um módulo de armazenamento superior ao do HDPE, o que não ocorre a temperatura ambiente. Isto é explicado pelo fato que, a baixas temperaturas, há um efeito de empacotamento das ramificações de pequena massa molar do LLDPE que promovem um maior ancoramento das cadeias poliméricas tornando este mais rígido, o que não ocorre para o HDPE, uma vez que este apresenta cadeia preferencialmente linear. Porém, no intervalo de temperatura em que ocorre a transição $\beta$ para o módulo de perda observa-se que o LLDPE passa a apresentar um módulo de armazenamento inferior ao HDPE, já que nessa temperatura as ramificações curtas da fase amorfa do primeiro ganham mobilidade diminuindo o ancoramento das cadeias principais e com isso diminuindo a rigidez do material.

As Figuras 6 e 7 apresentam as curvas de módulo de perda (E”) e módulo de armazenamento (E') para os
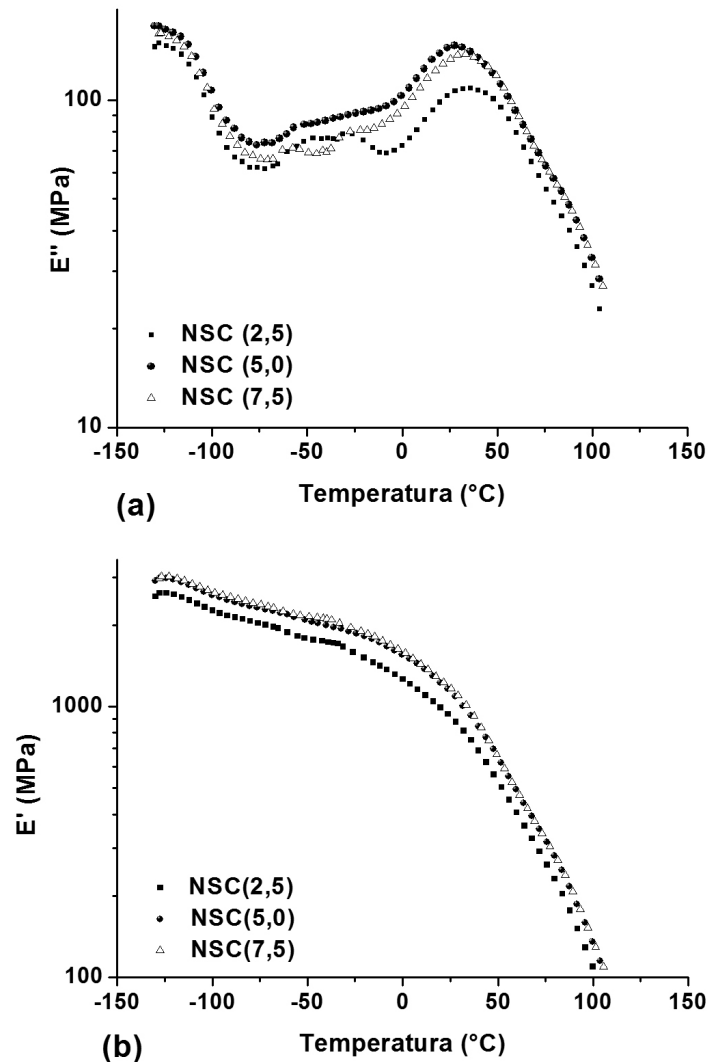

Figura 6. (a) Módulo de Perda (E") e (b) Módulo de Armazenamento (E') em função da temperatura dos nanocompósitos sem adição de agente compatibilizante. nanocompósitos sem adição de agente compatibilizante e compatibilizados com HDPE-g-MA, respectivamente.

Pode-se observar as relaxações $\alpha, \beta$ e $\gamma$ mesmo após a incorporação do argilomineral. Analisando o comportamento do módulo de armazenamento notase aumento com a incorporação da nanocarga até teor de $5 \%$ em massa. No entanto, com a adição de maiores quantidades de argila (com 7,5\% em massa de carga), com a formação de grandes tactóides e baixa interação polímero-carga, observou-se uma diminuição dos módulos de perda e armazenamento. Os valores das temperaturas de transição vítrea para a relaxação $\gamma$ foi de $-113,7{ }^{\circ} \mathrm{C}$ para NSC $(2,5),-114,5{ }^{\circ} \mathrm{C}$ para $\operatorname{NSC}(5,0)$ e $-106,5^{\circ} \mathrm{C}$ para $\mathrm{NSC}(7,5)$, valores superiores ao obtido para a blenda polimérica HDPE/LLDPE.

A adição de agente compatibilizante, com maior interação entre a carga e a matriz, diminuindo a mobilidade dos segmentos de cadeias poliméricas, possibilitou a diminuição da $\mathrm{Tg}$ dos nanocompósitos, sendo obtidos os valores de $-110,7{ }^{\circ} \mathrm{C}$ para $\mathrm{NC}(2,5),-108,3{ }^{\circ} \mathrm{C}$ para $\mathrm{NC}(5,0)$ e $-105,4^{\circ} \mathrm{C}$ para $\mathrm{NC}(7,5)$

\section{Temperatura de deflexão térmica}

Para a determinação da temperatura de deflexão térmica (HDT) dos corpos de prova obtidos, duas metodologias foram utilizadas: a determinação da HDT
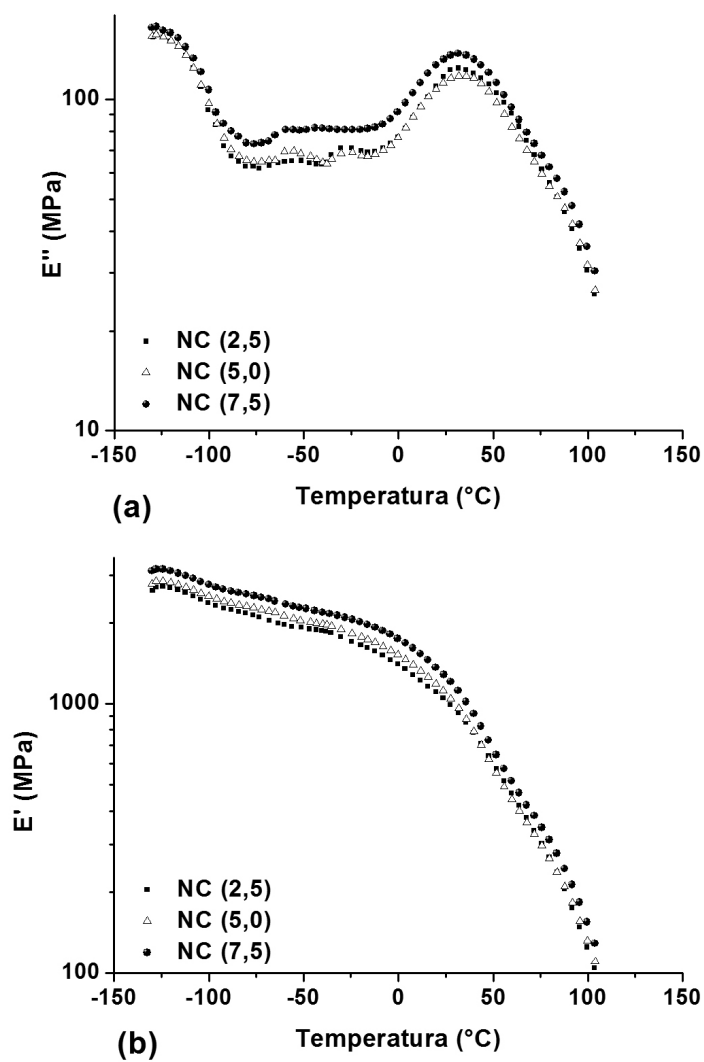

Figura 7. (a) Módulo de Perda (E”) e (b) Módulo de Armazenamento (E') em função da temperatura dos nanocompósitos compatibilizados com HDPE-g-MA. 
em um equipamento CEAST destinado a tal finalidade e a determinação da mesma através da análise dinâmicomecânica (DMA). Martins et al. ${ }^{[40]}$ estimaram a temperatura de deflexão térmica a partir de uma análise dinâmico-mecânica utilizando a técnica desenvolvida por Scobbo ${ }^{[41]}$. A norma ASTM D648 ${ }^{[42]}$ define a HDT como a temperatura na qual a deflexão central em um corpo de prova padronizado em flexão com três apoios atinge $0,25 \mathrm{~mm}$ sob uma tensão máxima aplicada $\sigma_{\max }$ de $0,46 \mathrm{ou}$ $1,82 \mathrm{MPa}$, tendo neste ensaio sido escolhido o primeiro valor. Utilizando a equação para a deflexão central de uma barra apoiada tem-se a relação entre o módulo elástico E, a tensão máxima e a deflexão ${ }^{[41]}$ :

$$
E=\sigma_{\max } L^{2} / 6 \Delta h
$$

onde L é a distância entre os apoios e h é a largura do corpo de prova que, considerando a disposição do corpo de prova neste ensaio, tomou-se o valor da espessura. Tomando $\mathrm{L}=18 \mathrm{~mm}$ e $\mathrm{h}=3,17 \mathrm{~mm}, \sigma_{\max }=0,46 \mathrm{MPa}$ e com amplitude de deformação imposta de $\Delta=0,03$ mm, obtém-se que E é aproximadamente 261,2 MPa. Assim, da curva de DMA do logaritmo do módulo de armazenamento E' versus Temperatura, a HDT é determinada como a temperatura em que $\mathrm{E}^{\prime}=261,2 \mathrm{MPa}$ ou $\log \mathrm{E}^{\prime}=8,42$.

A Tabela 1 apresenta os valores da temperatura de deflexão térmica obtida pelos dois métodos.

Como esperado, a adição da nanocarga aumentou a rigidez do material e com isso a temperatura em que os corpos de prova defletiram. Os nanocompósitos compatibilizados apresentaram propriedades superiores as que não receberam a adição do agente compatilizante. Foi observada também uma discrepância entre os valores para a temperatura de deflexão térmica determinada pelo aparelho HDT/VICAT e pela análise dinâmico-mecânica devido as diferentes naturezas dos ensaios, sendo que para este último as solicitações são cíclicas.

\section{Influência da ação do intemperismo nas propriedades de tração uniaxial}

A Figura 8 apresenta os resultados de módulo elástico dos materiais puros, da blenda polimérica e dos nanocompósitos submetidos aos diferentes tipos de envelhecimento. A Figura 9 apresenta a tensão de escoamento das amostras estudadas.

O HDPE, LLDPE e a blenda HDPE/LLDPE foram caracterizados por calorimetria exploratória diferencial (DSC) onde foi avaliado o primeiro ciclo de aquecimento e determinado o grau de cristalinidade, apresentados na Figura 10.

A análise do DSC das composições revelou que o envelhecimento em estufa de circulação de ar aumentou o grau de cristalinidade de todas as amostras, decorrente do alívio das tensões térmicas resultantes do processamento que permitiu a reordenação das moléculas.

As amostras submetidas ao tratamento em água também apresentaram aumento em suas propriedades, porém em menor intensidade, creditado a um maior grau de cristalinidade, adquirido em decorrência da temperatura da água, que estava a $60{ }^{\circ} \mathrm{C}$, sendo que nenhum comportamento plastificante foi identificado
Tabela 1. Temperatura de deflexão térmica (HDT) das composições estudadas.

\begin{tabular}{lcc}
\hline \multicolumn{1}{c}{ Amostra } & HDT $\left({ }^{\circ} \mathbf{C}\right)$ & HDT via DMA $\left({ }^{\circ} \mathbf{C}\right)$ \\
\hline HDPE & $81,0 \pm 0,7$ & 77,3 \\
LLDPE & $45,5 \pm 1,2$ & 47,2 \\
HDPE/LLDPE & $52,8 \pm 0,8$ & 69,7 \\
NSC $(2,5)$ & $59,5 \pm 0,8$ & 75,8 \\
NSC $(5,0)$ & $61,0 \pm 1,7$ & 82,1 \\
NSC $(7,5)$ & $61,5 \pm 1,9$ & 82,6 \\
NC $(2,5)$ & $61,4 \pm 1,0$ & 80,7 \\
NC $(5,0)$ & $67,4 \pm 0,2$ & 80,4 \\
NC $(7,5)$ & $80,7 \pm 2,3$ & 85,7 \\
\hline
\end{tabular}
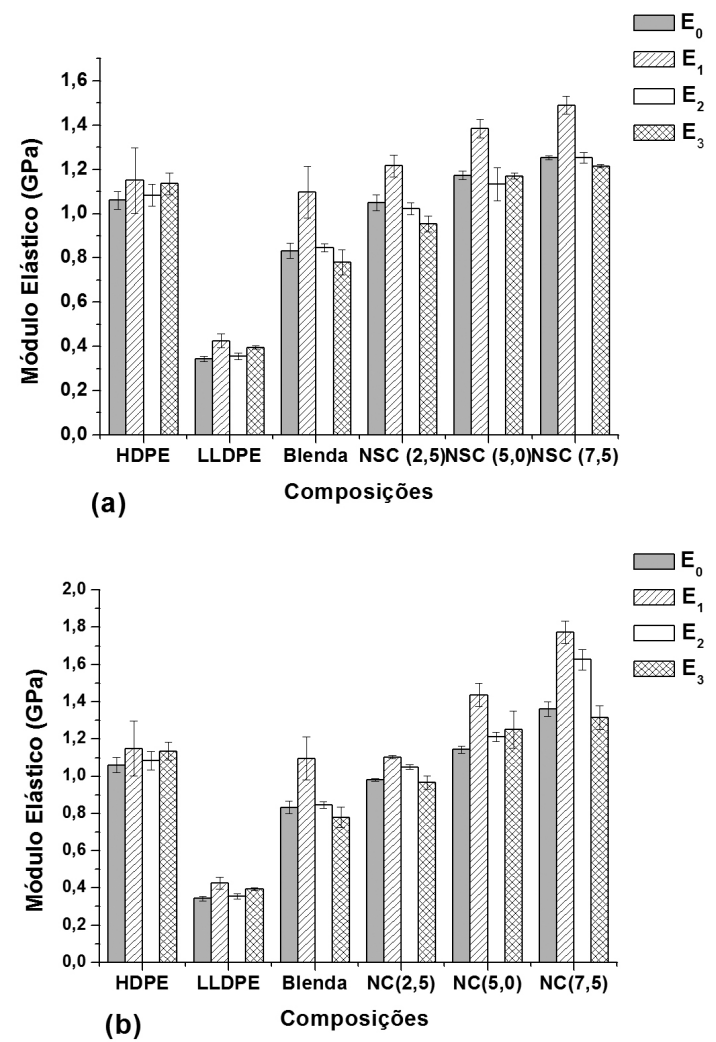

Figura 8. Módulo elástico das composições estudadas e submetidas aos diferentes tratamentos de envelhecimento (a) nanocompósitos sem adição de agente compatibilizante e (b) nanocompósitos compatibilizados com HDPE-g-MA.

devido à natureza hidrofóbica do material polimérico utilizado.

Em amostras submetidas ao envelhecimento acelerado em câmara de ultravioleta, observou-se uma rigidez levemente superior para o HDPE e LLDPE. Neste caso o período de exposição foi curto, estando as modificações observadas relacionadas a processos de reorganização molecular e estrutural, como indica Carrasco et al. ${ }^{[25]}$, que acompanharam o processo de envelhecimento artificial do polietileno de alta densidade e sugeriram que modificações químicas, como formação de grupos carbonila e vinílicos, que alterariam em maior 
intensidade as propriedades do polímero, predominariam somente em tempos de exposição superiores a 60 dias. Para o tempo de exposição utilizado não se observou indícios de ocorrência dos processos degradativos nos quais se formam compostos com muitas ramificações e com grau de cristalinidade superior aos sem tratamento. No entanto, pode ter ocorrido formação de grupos
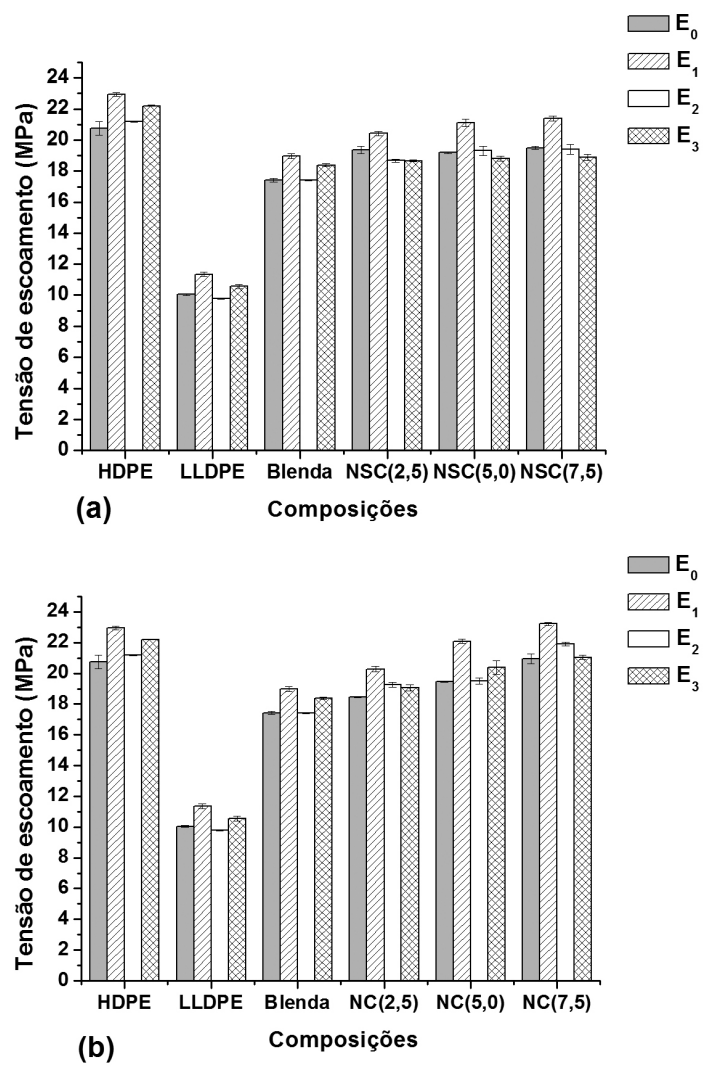

Figura 9. Tensão de escoamento das composições estudadas e submetidas aos diferentes tratamentos de envelhecimento (a) nanocompósitos sem adição de agente compatibilizante e (b) nanocompósitos compatibilizados com HDPE-g-MA.

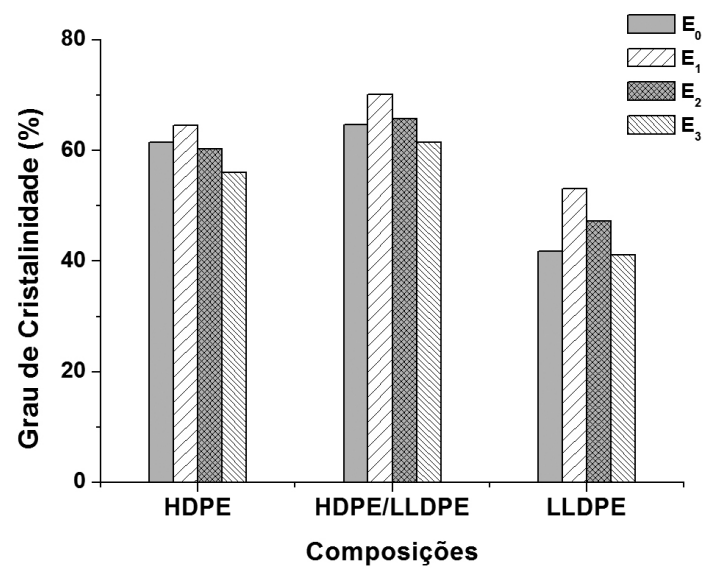

Figura 10. Grau de cristalinidade do HDPE, LLDPE e blenda HDPE/LLDPE em função dos diferentes tipos de envelhecimento. carbonilicos, peróxidos ou reticulação que podem aumentar a distância entre cadeias e, com isso, diminuir o grau de cristalinidade ${ }^{[25]}$.

De maneira geral observa-se que todos os nanocompósitos apresentaram propriedades mecânicas superiores à blenda HDPE/LLDPE referência. Comparando os nanocompósitos na condição $\mathrm{E}_{0}$ (referência), observa-se que para teores de até 5,0\% em massa de argilomineral os nanocompósitos sem adição de agente compatibilizante apresentaram propriedades mecânicas levemente superiores aos nanocompósitos compatibilizados com HDPE-gMA. Este comportamento também foi observado por Spencer et al. ${ }^{[12]}$ ao estudar blendas HDPE-g-MA/HDPE, devido à menor cristalinidade do primeiro em relação ao segundo. Analisando as composições com 7,5\% em massa de OMMT, observa-se que a composição $\mathrm{NC}(7,5)$ apresentou módulo elástico e tensão de escoamento superior a composição $\operatorname{NSC}(7,5)$, mostrando que o maior teor de nanocarga empregado suplantou as perdas devido à adição do agente compatibilizante.

Para o tratamento em estufa por três dias, identificado como $\mathrm{E}_{1}$, observa-se um aumento no módulo elástico e na tensão de escoamento para todas as amostras, devido ao maior grau de cristalinidade conferido a estas após este tratamento e não a processos termo-oxidativos, já que o tempo de exposição foi reduzido. As amostras submetidas ao tratamento em água $\left(\mathrm{E}_{2}\right)$ também apresentaram aumento em suas propriedades, porém em menor intensidade, também devido a um maior grau de cristalinidade adquirido em decorrência da temperatura da água, que estava a $60{ }^{\circ} \mathrm{C}$ e nenhum comportamento plastificante foi identificado devido à natureza hidrofóbica do material polimérico utilizado.

Com relação aos corpos de prova submetidos ao procedimento de envelhecimento acelerado em câmara de ultravioleta $\left(\mathrm{E}_{3}\right)$, observou-se nestes uma rigidez levemente superior às amostras referências. Neste caso o período de exposição foi curto, estando as modificações observadas relacionadas a processos de reorganização molecular e estrutural, como indica Carrasco et al. ${ }^{[23]}$, que acompanharam o processo de envelhecimento artificial do polietileno de alta densidade e sugeriram que modificações químicas, como formação de grupos carbonila e vinílicos, que alterariam em maior intensidade as propriedades do polímero, predominariam somente em tempos de exposição superiores a 60 dias.

\section{Conclusão}

$\mathrm{O}$ agente compatibilizante escolhido, HDPE-g-MA, mostrou-se adequado para o sistema utilizado, com formação de estrutura predominantemente intercalada. Os nanocompósitos apresentaram propriedades térmicas e mecânicas superiores à blenda HDPE/LLDPE. A adição de HDPE-g-MA possibilitou maior interação entre a carga e a matriz, e ocorreu diminuição da mobilidade dos segmentos de cadeias poliméricas e conseqüente diminuição da $\mathrm{Tg}$ dos nanocompósitos.

As diferentes condições de intemperismo as quais os materiais foram submetidos influenciaram o comportamento mecânico dos nanocompósitos de 
blenda HDPE/LLDPE. O tratamento realizado em estufa com circulação forçada de ar proporcionou o alívio das tensões residuais decorrentes do processamento e, como consequência, o aumento no grau de cristalinidade. O tratamento em água também ocasionou aumento do módulo elástico, principalmente por gerar condições propícias para alívio de tensões residuais. $\mathrm{O}$ tratamento em câmara de envelhecimento acelerado com exposição simultânea à luz ultravioleta e água também afetou as propriedades mecânicas, principalmente devido a formação de carbonilas, de pequena massa molar, que diminuem o grau de cristalinidade e o módulo elástico dos nanocompósitos.

\section{Agradecimentos}

Os autores agradecem ao CNPq e a FAPESP pelo apoio financeiro.

\section{Referências Bibliográficas}

1. Bower, D. I. - "An Introduction to Polymer Physics", Cambridge University Press, New York (2002). http:// dx.doi.org/10.1017/CBO9780511801280

2. Coutinho, F. M. B.; Mello, I. L. \& De Santa Maria, L. C. - Polímeros, 13, p.1 (2003).

3. Munaro, M. \& Akcelrud, L. - Polym. Degrad. Stab., 93, p.43 (2008). http://dx.doi.org/10.1016/j. polymdegradstab.2007.10.017

4. Al-Salem, S. M. - Materials \& Design, 30, p.1729 (2009). http://dx.doi.org/10.1016/j.matdes.2008.07.049

5. Gopakumar, T. G.; Lee, J. A.; Kontopoulou, M. \& Parent, J, S. - Polymer, 43, p.5483 (2002). http://dx.doi.org/10.1016/ S0032-3861(02)00403-2

6. Hotta, S. \& Paul, D. R. - Polymer, 45, p.7639 (2004). http:// dx.doi.org/10.1016/j.polymer.2004.08.059

7. Brito, G. F.; Oliveira, A. D; Araújo, E. M.; Melo, T. J. A.; Barbosa, R. \& Ito, E. N. - Polímeros, 18, p.170 (2008). http://dx.doi.org/10.1590/S0104-14282008000200015

8. Barbosa, R.; Araújo, E. M.; Melo, T. J. A. \& Ito, E. N. - Polímeros, 17, p.104 (2007).

9. Jérôme, R.; Garces, J.M.; Sun, T.; Dubois, P. \& Alexandre, M. - Polymer, 43, p.2123 (2002).

10. Morgan, A. B. \& Gilman, J. W. - J. Appl. Polym Sci, 87, p.1329 (2003). http://dx.doi.org/10.1002/app.11884

11. Chrissopoulou, K.; Altintzi, I.; Anastasiadis, S. H.; Giannelis, E. P.; Pitsikalis, M.; Hadjichristidis, N. \& Theophilou, N. - Polymer, 46, p.12440 (2005). http:// dx.doi.org/10.1016/j.polymer.2005.10.106

12. Spencer, M. W.; Cui, L.; Yoo, Y. \& Paul, D. R. - Polymer, 51, p.1056 (2010). http://dx.doi.org/10.1016/j. polymer.2009.12.047

13. Wang, K. H.; Choi, M. H.; Koo, C. M.; Choi, Y. S. \& Chung, I. J. - Polymer, 42, p.9819 (2001). http://dx.doi. org/10.1016/S0032-3861(01)00509-2

14. Rabello, M. S. - "Aditivação de Polímeros", Ed. Artliber, São Paulo (2007).

15. Chatterjee, N.; Basu, S.; Palit, S. K. \& Maiti, M. M. - J. Polym. Sci. Part B: Phys., 33, p.1705 (1995). http://dx.doi. org/10.1002/polb.1995.090331201
16. Allen, N. S.; Edge, M.; Corrales, T.; Shah, M.; Holdsworth, D.; Catalina, F.; Peinado, C. \& Collar, E. P. - Polymer, 37, p.2323 (1996). http://dx.doi.org/10.1016/0032-3861(96)85342-0

17. Zhao, Y.; Luo, Y. \& Jiang, B. - J. Appl. Polym Sci, 50, p.1797 (1993). http://dx.doi.org/10.1002/app.1993.070501015

18. Rabello, M. S. \& White, J. R. - Polymer, 38, p.6379 (1997). http://dx.doi.org/10.1016/S0032-3861(97)00213-9

19. Khabbaz, F.; Albertsson, A. C. \& Karlsson, S. - Polym. Degrad. Stab., 63, p.127 (1999). http://dx.doi.org/10.1016/ S0141-3910(98)00082-2

20. Kostoski, D. \& Stojanovic, Z. - Polym. Degrad. Stab., 47, p.353 (1995). http://dx.doi.org/10.1016/01413910(94)00126-X

21. Terselius, B.; Gedde, U. W. \& Jansson, J. F. - Polym. Eng. Sci., 22, p.422 (1982). http://dx.doi.org/10.1002/ pen.760220706

22. Ungar, G. \& Keller, A. - Polymer, 21, p.1273 (1980). http:// dx.doi.org/10.1016/0032-3861(80)90192-5

23. Yang, R., Li, Y.\& Yu, J. - Polym. Degrad. Stab., 88, p.168 (2005). http://dx.doi.org/10.1016/j.polymdegradstab.2003.12.005

24. Gulmine, J. V.; Janissek, P. R.; Heise, H. M. \& Akcelrud, H. M. - Polym. Degrad. Stab., 79, p.385 (2003). http://dx.doi. org/10.1016/S0141-3910(02)00338-5

25. Carrasco, F.; Pages, P.; Pascual, S. \& Colom, X. - Eur. Polym. J., 37, p.1457 (2001). http://dx.doi.org/10.1016/ S0014-3057(00)00251-2

26. Allen , N. S.; Edge, M.; Corrales, T.; Childs, A.; Liauw, C. M.; Catalina, F., Peinado, C.; Minihand, A. \& Aldcroft, D. - Polym. Degrad. Stab., 61, p.183 (1998). http://dx.doi. org/10.1016/S0141-3910(97)00114-6

27. Grigoriadou, I.; Paraskevopoulos, K. M.; Chrissafis, K.; Pavlidou, E. \& Stamkopoulos - Polym. Degrad. Stab., 96, p.151 (2011). http://dx.doi.org/10.1016/j. polymdegradstab.2010.10.001

28. Morlat-Therias, S.; Fanton, E.; Gardette, J.; Dintcheva, N. T.; La Mantia, F. P. \& Malatesta, V. - Polym. Degrad. Stab., 93, p.1776 (2008). http://dx.doi.org/10.1016/j. polymdegradstab.2008.07.031

29. Nitta, K. H. \& Tanaka, A. - Polymer, 42, p.1219 (2001). http://dx.doi.org/10.1016/S0032-3861(00)00418-3

30. Kajiyama, T.; Okada, T.; Sakoda, A. \& Takayanagi, M. - J. Macromol. Sci. B: Phys., 7, p.583 (1973). http://dx.doi. org/10.1080/00222347308207887

31. Kyu, T.; Yasuda, N.; Suehiro, S.; Nomura, S. \& Kawai, H. - Polym. J., 8, p.565 (1976). http://dx.doi.org/10.1295/ polymj.8.565

32. Takayanagi, M.; Aramaki, T.; Yoshino, M. \& Hoashi, K. - J. Polym. Sci., 46, p.531 (1960). http://dx.doi.org/10.1002/ pol.1960.1204614824

33. Pechhold, W.; Eisele, U. \& Knauss, G. - Coll. Polym. Sci., 196, p.27 (1964).

34. Moore, R. S. \& Matsuoka, S. - J. Polym. Sci. C: Polym. Symposia, 5, p.163 (1964). http://dx.doi.org/10.1002/ polc.5070050115

35. Kline, D. E.; Sauer, J. A. \& Woodward, A. E. - J. Polym. Sci., 22, p.455 (1956). http://dx.doi.org/10.1002/ pol.1956.1202210211

36. Popli, R.; Glotin, M.; Mandelkern, L. \& Benson, R. S. - J. Polym. Sci.: Polym. Phys. Ed., 22, p.407 (1984). http:// dx.doi.org/10.1002/pol.1984.180220306 
37. Wada, Y.; Tsuge, K.; Arisawa, K.; Shisa, K.; Hotta, Y.; Hayakawa, R. \& Nishi, T. - J. Polym. Sci. C , 15, p.101 (1966). http://dx.doi.org/10.1002/polc.5070150110

38. Fischer, E. W.; Peterlin, A. - Die Makromolekulare Chemie, 74, p.1 (1964). http://dx.doi.org/10.1002/macp.1964.020740101

39. Wollbourn, A. H. - Trans. Faraday Soc., 54, p.717 (1968).

40. Martins, C. G.; Larocca, N. M.; Paul, D. R. \& Pessan, L. A. - Polymer, 50, p.1743 (2009). http://dx.doi.org/10.1016/j. polymer.2009.01.059

41. Scobbo, J. J. In: Paul, D. R. \& Bucknall, C. B. - "Polymer blends", Academic Press Inc, New York, v. 1, p.335 (2000).
42. American Society for Testing and Materiais - "ASTM D648-07: Standart Test Method for Deflection Temperature of Plastics Under Flexural Load in the Edgwise Position", ASTM (2007).

43. American Society for Testing and Materiais - "ASTM G155-00: Standart Practice for Operating Xenor Arc Light Apparatus for Exposure of Non-Metallic Materials", $\operatorname{ASTM}(2000)$.

Enviado: $29 / 05 / 12$

Reenviado: $29 / 06 / 12$

Aceito: $16 / 08 / 12$ 